\title{
Prevalence of Retinal Vein Occlusions and Estimated Cerebrospinal Fluid Pressure: The Kailuan Eye Study
}

\author{
Qian Wang ${ }^{1} *$ \\ Jingyan Yang $\mathbb{D}^{1, *}$ \\ Jost B Jonas $\mathbb{( D}^{2}$ \\ Xuehui Shi ${ }^{1}$ \\ Shouling $\mathrm{Wu}^{3}$ \\ Shuohua Chen ${ }^{4}$ \\ Yanni Yan' \\ Wenjia Zhou' \\ Li Dong' \\ Wenbin Wei ${ }^{1}$ \\ Ya Xing Wang ${ }^{5}$
}

'Beijing Tongren Eye Center, Beijing Key Laboratory of Intraocular Tumor Diagnosis and Treatment, Beijing Ophthalmology \& Visual Sciences Key Lab, Medical Artificial Intelligence Research and Verification Laboratory of the Ministry of Industry and Information Technology, Beijing Tongren Hospital, Capital Medical University, Beijing, People's Republic of China; ${ }^{2}$ Department of Ophthalmology, Medical Faculty Mannheim of the Ruprecht-Karis-University, Mannheim, Germany; ${ }^{3}$ Cardiology Department, Kailuan General Hospital, Tangshan, People's Republic of China; ${ }^{4}$ Health Care Center, Kailuan Group, Tangshan, People's Republic of China; ${ }^{5}$ Beijing Institute of Ophthalmology and Beijing Ophthalmology and Visual Science Key Lab, Beijing Tongren Eye Center, Beijing Tongren Hospital, Capital Medical University, Beijing, People's Republic of China

*These authors contributed equally to this work

Correspondence: Wenbin Wei

Beijing Tongren Eye Center, Beijing Tongren Hospital, Capital Medical University, I Dong Jiao Min Xiang, Dong Cheng District, Beijing, 100730, People's Republic of China

Tel +86-10-58269516

Fax +86- I0-65I2 5617

Email weiwenbintr@।63.com
Purpose: To investigate the prevalence of retinal vein occlusions (RVOs) and associated factors in a Chinese population.

Patients and Methods: The cross-sectional community-based Kailuan Eye Study included individuals who participated in the Kailuan Study. RVOs were diagnosed on the fundus photographs. Estimated cerebrospinal fluid pressure (eCSFP) was calculated as "eCSFP $=0.44 *$ Body Mass Index +0.16 *Diastolic Blood Pressure-0.18*Age".

Results: The study included 12,499 participants with a mean age of $52.9 \pm 13.1$ years. The overall prevalence of RVO was $120 / 12,499$ or $0.96 \%$, with branch RVOs observed in 116 / 12,499 individuals and central RVOs in 4/12,499 individuals. RVOs started at the optic disc in 19 participants (15.8\% of all RVOs), and in 101 (84.2\%) individuals arterio-venous crossings outside the optic disc. In multivariable analysis, a higher RVO prevalence was associated with older age $(P<0.001)$, higher eCSFP $(P<0.001)$, and higher fasting serum glucose concentration $(P<0.001)$. Differentiating between RVOs at arterio-venous crossings and RVOs at the optic disc revealed that the prevalence of both RVO types was associated with higher eCSFP ( $P<0.001$ and $P=0.004$, respectively) after adjusting for age and fasting serum glucose concentration.

Conclusion: In this adult Chinese population recruited on a community basis, the prevalence of any RVO (mean: 0.96) was associated with older age, higher eCSFP and higher fasting serum glucose concentration. Higher eCSFP may play an etiologic role in RVOs.

Keywords: retinal vein occlusion, blood pressure, arterial hypertension, glaucoma, intraocular pressure, cerebrospinal fluid pressure

\section{Introduction}

Among retinal vascular diseases, retinal vein occlusions (RVOs) are one of the most common causes of visual impairment, second only to diabetic retinopathy. ${ }^{1,2}$ Among other sequelae, RVOs can lead to macular edema and vitreous hemorrhage markedly reducing central visual acuity. ${ }^{3-5}$ A recent analysis by Rogers and colleagues revealed that approximately 16.4 million adults worldwide are affected by RVOs. ${ }^{1}$ The RVO prevalence as reported in previous investigations ranges from $0.26 \%$ to $3.39 \%$, with the associated factors of older age and a higher prevalence of systemic diseases, such as arterial hypertension, diabetes mellitus, arteriosclerosis, and hyperlipidemia. 1,2,6,7 These findings were obtained mostly in studies focused on the ophthalmological part with relatively little data of major systemic diseases available, and some of the investigations were based on relatively small study 
populations. $^{6-11}$ In addition, the previous studies did not generally differentiate between RVOs originating from the optic disc and those starting at a arterio-venous crossing outside of the optic nerve head. With respect to the pathogenesis of RVOs, several risk factors and mechanisms have been discussed to be involved in the etiology of RVOs. $^{3,6-11}$ It includes arterial hypertension and glaucoma, with glaucoma-related morphological changes in the lamina cribrosa leading potentially to an increased trans-lamina cribrosa outflow resistance for the venous blood. Recently, an increased cerebrospinal fluid pressure (CSFP) has been considered to be etiologically associated with the incidence of RVOs. ${ }^{12}$ We therefore conducted this study to investigate the prevalence of RVOs as a whole and stratified by the location of their starting point and to assess a potential relationship between the CSFP and the prevalence of RVOs in a relatively large study population which had undergone an intensive internal medical examination.

\section{Patients and Methods}

The cross-sectional community-based Kailuan Eye Study consisted of the participants of the longitudinal Kailuan Study. The study protocol was approved by the Medical Ethics Committee of the Beijing Tongren Hospital, and a written informed consent was obtained from every study participant, and all examinations were conducted in accordance with the Declaration of Helsinki. Kailuan, a center of the coal mining industry, is part of the city of Tangshan which is located about $150 \mathrm{~km}$ southeast of Beijing. The study population consisted of employees and retirees and their family members of a coal mining company and its associated industries and businesses. The Kailuan Study population included at baseline a cohort of 101,510 participants with an age between 18 and 111 years, who were biennially re-examined. ${ }^{13-15}$ Using an examination unit-based cluster random sampling method, we randomly selected a sample of 14,440 subjects out of the Kailuan cohort to participate in the Kailuan Eye Study. Out of these 14,440 individuals, $12,499(86.6 \%)$ participants underwent ocular fundus photography of both eyes that were considered gradable for the assessment of the presence of RVOs. The individuals included in the present study and those Kailuan Study participants who were not included in this study did not differ significantly in gender (men/women: 8483/ 4016 (67.9\%/32.1\%) versus 1352/589 (69.7\%/30.3\%); $P=0.12$ ). The individuals included in the present study were significantly younger than those who were not included in this study $(52.93 \pm 13.07$ years versus 60.84 \pm 12.36 years; $P<0.001)$.

The first part of the study examinations consisted of an interview assessing socioeconomic parameters (eg, educational level), lifestyle variables (eg, physical activity, alcohol consumption), and major internal medical disorders. We measured body weight and height, waist and hip circumference, heart rate, and blood pressure. Biochemical examinations of blood samples obtained under fasting conditions assessed the serum concentrations of glucose, hemoglobin, high-density and low-density lipoprotein cholesterol, triglycerides and total cholesterol, albumin, uric acid, high-sensitivity C-reactive protein, glutamate pyruvate transaminase, total bilirubin, direct bilirubin, and urea. Additionally, we performed a blood cell platelet count.

Using the derived formula, we estimated the cerebrospinal fluid pressure (eCSFP): “eCSFP $[\mathrm{mmHg}]=0.44 *$ Body Mass Index $[\mathrm{kg} / \mathrm{m} 2]+0.16 *$ Diastolic Blood Pressure [mmHg]-0.18* Age [Years]". ${ }^{16}$

Diabetes mellitus was defined by a fasting serum glucose concentration of $\geq 7.0 \mathrm{mM}$ during the 12-year followup period, or a history of diabetes or of anti-diabetic medication. Criteria for the diagnosis of hypertension were a systolic blood pressure of equal to or more than $140 \mathrm{mmHg}$, a diastolic blood pressure of equal to or more than $90 \mathrm{mmHg}$, and/or current anti-hypertensive treatment.

The series of ocular examinations consisted of the assessment of visual acuity, slit-lamp-based biomicroscopy, and ocular biometry by optical low-coherence reflectometry (Lenstar 900 Optical Biometer; Haag-Streit, Koeniz, Switzerland). We measured central corneal thickness, anterior chamber depth, lens thickness and axial length. We took two $45^{\circ}$ fundus photographs centered on the optic disc and on the macula (non-mydriatic fundus camera; CR6-45NM; Cannon, Inc., Ōsta, Tokyo, Japan).

In a masked manner, we assessed the presence of RVOs on the fundus photographs of both eyes of the study participants. We differentiated RVOs into central retinal vein occlusions (CRVOs) (including hemi-central RVOs) and branch retinal vein occlusions (BRVOs). Retinal and optic disc edema, retinal hemorrhages and venous dilation were the characteristics of a recent CRVO, while occluded and sheathed retinal veins or vascular anastomoses at the optic disc were typical for long standing CRVOs. BRVOs showed localized changes in the form of retinal edema and hemorrhages, intraretinal 
microvascular abnormalities or anastomotic vessels, and venous dilatation or venous sheathing within a retinal sector corresponding to the obstructed vein. ${ }^{17,18}$ According to the location of the BRVO, we differentiated between RVOs starting at the optic disc, and RVOs beginning at an arterio-venous crossing outside of the optic disc. The fundus photographs were examined first by an experienced ophthalmologist (Q.W.), and all fundus images of eyes with a suspected RVO were re-assessed by a panel of ophthalmologists (Q.W., Y.X.W., X.H.S., J.B.J., W.B.W.).

A statistical software program (SPSS for Mac, version 25.0; IBM/SPSS, Chicago, IL, USA) was used for statistical analysis. We described the primary results as mean \pm standard deviation for continuous variables, and as percentage and 95\% confidence interval (CI) for categorical variables. We applied the chi-square test for proportions or the Student's $t$-test to compare the baseline characteristics of the individuals with versus without RVOs, and we used logistic regression models to calculate the odds ratios (ORs) and their 95\% CIs of each factor associated with RVOs.

\section{Results}

The Kailuan Eye Study primarily included 14,440 individuals (9835 (68.1\%) men)) with a mean age of $54.0 \pm 13.3$ years (median: 56.0 years; range: $20-110$ years). Since the fundus photographs of 1941 (13.4\%) individuals could not be evaluated due to insufficient quality of the fundus photographs for one eye or both eyes, the present study eventually included $12,499(86.6 \%)$ participants (8483 men $(67.9 \%))$. These were significantly younger (mean age: $52.9 \pm 13.17$ years; median: 55 years; range: $20-110$ years; $P<0.001$ ) than the group of individuals without assessable fundus photographs, while both groups did not differ significantly in gender $(P=0.12)$.

The overall prevalence of any RVO was $120 / 12,499$ or $0.96 \%$ (95\% CI: $0.80,1.15)$, with branch retinal vein occlusions (BRVO) observed in $116 / 12,499$ or $0.93 \%$ (95\% CI: $0.77,1.11)$ and central retinal vein occlusions (CRVO) in $4 / 12,499$ or $0.032 \%$ (95\% CI: $0.012,0.085)$. Bilateral BRVOs were present in three participants (3 or $2.5 \%$ of all individuals with RVOs), but none had a bilateral CRVO. In 19 participants $(15.8 \%$ of all individuals with RVOs), the vein occlusion occurred in the optic disc and in $101(84.2 \%)$ the retinal changes started at a arterio-venous crossing outside of the optic disc.

Increasing with older age, the overall prevalence of any RVO was $0.13 \%(95 \% \mathrm{CI}: 0.04,0.40)$ in those aged $<40$ years, $0.68 \%$ (95\% CI: $0.46,1.02)$ in those aged 50-59 years, and $3.31 \%(95 \% \mathrm{CI}: 1.26,8.67)$ in those $\geq 80$ years (Table 1). In univariable analysis, the participants with RVOs compared with the participants without vein occlusions had a significantly (all $P<0.05$ ) higher age, higher prevalence of male gender, shallower anterior chamber, thicker lens, shorter axial length, higher systolic and diastolic blood pressure, higher BMI, higher fasting blood glucose, higher uric acid serum concentration, higher waist-hip ratio, and higher prevalence of diabetes and hypertension (Table 2).

In the multivariable binary analysis, the RVO prevalence was the dependent parameter. Independent parameters were those parameters which were correlated ( $P$-value $\leq 0.10)$ with the RVO prevalence in the univariable analysis (Table 2). We first removed the parameters of lens thickness and anterior chamber depth from the list of independent parameters due to their collinearity with axial

Table I Age-Specific Prevalence (Mean and 95\% Confidence Intervals) of Retinal Vein Occlusions (RVOs), Stratified into RVOs Originating at the Optic Nerve Head and RVOs Originating at Arterio-Venous Crossings Outside of the Optic Nerve Head

\begin{tabular}{|l|c|c|c|}
\hline \multirow{2}{*}{ Age (Years) } & \multicolumn{3}{|c|}{ Percentage (95\% Confidence Intervals) (Number of Effected Individuals) } \\
\cline { 2 - 4 } & $\begin{array}{c}\text { RVO Originating at the Optic } \\
\text { Nerve Head }\end{array}$ & $\begin{array}{c}\text { RVO Originating at Arterio-Venous Crossings Outside of } \\
\text { the Optic Nerve Head }\end{array}$ & Total \\
\hline$\leq 39$ & $0.04(0.006,0.31)(1)$ & $0.09(0.022,0.35)(2)$ & $0.13(0.04,0.40)(3)$ \\
$40-49$ & $0.09(0.02,0.37)(2)$ & $0.50(0.28,0.91)(I I)$ & $0.60(0.35,1.03)(13)$ \\
$50-59$ & $0(0)$ & $0.68(0.46,1.02)(24)$ & $0.68(0.46,1.02)(24)$ \\
$60-69$ & $0.34(0.19,0.60)(12)$ & $1.41(1.07,1.86)(50)$ & $1.75(1.37,2.24)(62)$ \\
$70-79$ & $0.49(0.18,1.29)(4)$ & $1.22(0.66,2.25)(10)$ & $1.70(1.01,2.86)(14)$ \\
$\geq 80$ & $0(0)$ & $3.31(1.26,8.67)(4)$ & $3.31(1.26,8.67)(4)$ \\
Overall & $0.15(0.097,0.24)(19)$ & $0.81(0.67,0.98)(101)$ & - \\
\hline
\end{tabular}


Table 2 Differences Between the Group of Participants with Retinal Vein Occlusions Compared with the Group of Participants without Retinal Vein Occlusions in the Kailuan Eye Study

\begin{tabular}{|c|c|c|c|}
\hline & Retinal Vein Occlusions & No Retinal Vein Occlusions & $P$-value \\
\hline $\mathrm{n}$ & $120(0.96 \%)$ & $12,379(99.04 \%)$ & \\
\hline Age (Years) & $61.2 \pm 9.8$ & $52.9 \pm 13.1$ & $<0.001$ \\
\hline Gender (men) & $97(80.8 \%)$ & $8386(67.8 \%)$ & 0.003 \\
\hline History of diabetes mellitus & $36(31.6 \%)$ & $2297(19.8 \%)$ & 0.002 \\
\hline History of hypertension & $85(74.6 \%)$ & $5308(45.7 \%)$ & $<0.001$ \\
\hline Mean systolic blood pressure $(\mathrm{mmHg})$ & $150.4 \pm 22.0$ & $137.2 \pm 18.9$ & $<0.001$ \\
\hline Mean diastolic blood pressure $(\mathrm{mmHg})$ & $85.4 \pm 11.8$ & $79.9 \pm 10.3$ & $<0.001$ \\
\hline Mean fasting blood glucose $(\mathrm{mmol} / \mathrm{L})$ & $7.19 \pm 3.27$ & $6.06 \pm 1.96$ & $<0.001$ \\
\hline Mean heart rate (beats/minute) & $75.6 \pm 12.0$ & $75.6 \pm 11.2$ & 0.99 \\
\hline Mean waist-hip ratio & $0.92 \pm 0.09$ & $0.89 \pm 0.08$ & $<0.001$ \\
\hline Mean body mass index $\left(\mathrm{kg} / \mathrm{m}^{2}\right)$ & $26.1 \pm 3.7$ & $25.0 \pm 3.4$ & 0.001 \\
\hline Mean high-density lipoprotein cholesterol concentration ( $\mathrm{mmol} / \mathrm{L})$ & $1.46 \pm 0.49$ & $1.54 \pm 0.60$ & 0.18 \\
\hline Mean low-density lipoprotein cholesterol concentration (mmol/L) & $2.82 \pm 0.80$ & $2.97 \pm 0.92$ & 0.08 \\
\hline Mean triglyceride $(\mathrm{mmol} / \mathrm{L})$ & $2.26 \pm 1.72$ & $2.10 \pm 2.26$ & 0.46 \\
\hline Mean total cholesterol concentration $(\mathrm{mmol} / \mathrm{L})$ & $4.40 \pm 1.72$ & $4.63 \pm 1.97$ & 0.21 \\
\hline Mean uric acid concentration $(\mu \mathrm{mol} / \mathrm{L})$ & $348 \pm 104$ & $326 \pm 88$ & 0.03 \\
\hline Mean hypersensitive C-reactive protein concentration $(\mathrm{mg} / \mathrm{L})$ & $1.81 \pm 2.43$ & $1.98 \pm 7.22$ & 0.80 \\
\hline Mean total protein concentration $(\mathrm{g} / \mathrm{L})$ & $74.1 \pm 5.4$ & $73.2 \pm 5.1$ & 0.10 \\
\hline Mean albumin $(g / L)$ & $46.3 \pm 3.5$ & $47.4 \pm 7.3$ & 0.13 \\
\hline Mean glutamate pyruvate transaminase $(\mathrm{U} / \mathrm{L})$ & $23.2 \pm 15.6$ & $23.7 \pm 21.9$ & 0.79 \\
\hline Mean total bilirubin $(\mu \mathrm{mol} / \mathrm{L})$ & $15.7 \pm 6.1$ & $15.2 \pm 12.0$ & 0.67 \\
\hline Creatinine $(\mu \mathrm{mol} / \mathrm{L})$ & $77.2 \pm 20.2$ & $72.7 \pm 27.8$ & 0.08 \\
\hline Mean urea $(\mathrm{mmol} / \mathrm{L})$ & $5.88 \pm 1.56$ & $5.65 \pm 5.99$ & 0.68 \\
\hline Mean urine $\mathrm{pH}$ & $5.93 \pm 0.68$ & $5.87 \pm 0.62$ & 0.38 \\
\hline Mean red blood cell count $\left(10^{12} / \mathrm{L}\right)$ & $4.94 \pm 0.46$ & $4.88 \pm 0.47$ & 0.22 \\
\hline Mean white blood cell count $\left(10^{9} / \mathrm{L}\right)$ & $6.10 \pm 1.93$ & $5.93 \pm 1.77$ & 0.33 \\
\hline Mean blood platelet count (109/L) & $228 \pm 54$ & $238 \pm 57$ & 0.07 \\
\hline Mean hemoglobin $(\mathrm{g} / \mathrm{L})$ & $148.2 \pm 19.3$ & $148.4 \pm 16.0$ & 0.89 \\
\hline Mean neutrophil cell count $\left(10^{9} / \mathrm{L}\right)$ & $3.87 \pm 1.39$ & $3.73 \pm 2.08$ & 0.47 \\
\hline Central cornea thickness $(\mu \mathrm{m})$ & $535 \pm 29$ & $537 \pm 33$ & 0.60 \\
\hline Anterior chamber depth $(\mathrm{mm})$ & $2.62 \pm 0.39$ & $2.73 \pm 0.45$ & 0.046 \\
\hline Lens thickness $(\mathrm{mm})$ & $4.43 \pm 0.38$ & $4.26 \pm 0.61$ & 0.035 \\
\hline Axial length $(\mathrm{mm})$ & $23.41 \pm 0.91$ & $23.71 \pm 1.28$ & 0.01 \\
\hline
\end{tabular}

length, diastolic blood pressure due to its collinearity with systolic blood pressure, and low-density lipoprotein serum concentration due to its collinearity with fasting blood glucose serum concentration. Due to a lack of statistical significance, we then dropped step-by-step the parameters of axial length $(P=0.95)$, the serum concentration of uric acid $(P=0.63)$, total protein $(P=0.92)$ and creatinine ( $P=0.62)$, waist-hip ratio $(P=0.44)$, gender $(P=0.94)$, history of diabetes mellitus $(P=0.15)$ and arterial hypertension $(P=0.51)$, and blood platelet count $(P=0.54)$. In the final model, a higher RVO prevalence correlated with older age $(P<0.001)$, higher systolic blood pressure ( $P=0.001)$, higher body mass index (BMI) $(P=0.007)$ and higher fasting blood glucose serum concentration
$(P=0.002)$ (Table 3). If the parameters of blood pressure and BMI were replaced by the estimated cerebrospinal fluid pressure (eCSFP) parameter (which comprised blood pressure and BMI), a higher RVO prevalence was associated in the final model with a higher eCSFP $(P<0.001)$, older age $(P<0.001)$, and higher fasting blood glucose serum concentration $(P<0.001)$ (Table 3$)$.

In univariate logistic analysis, as compared with participants without RVOs, participants with RVOs originating at the optic disc had a significantly (all $P<0.05$ ) older age, shallower anterior chamber, lower prevalence of arterial hypertension, higher systolic and diastolic blood pressure and higher uric acid serum concentration (Table 4). In the multivariable logistic regression analysis, we removed 
Table 3 Associations (Multivariable Logistic Regression Analysis) Between the Prevalence of Retinal Vein Occlusions and Ocular and Systemic Parameters in the Kailuan Eye Study

\begin{tabular}{|l|c|c|c|}
\hline Parameters & Odds Ratio & 95\% Confidence Interval for OR & P-value \\
\hline Age & 1.05 & $1.03,1.07$ & $<0.001$ \\
\hline Systolic blood pressure & 1.02 & $1.01,1.03$ & 0.001 \\
\hline Body mass index & 1.08 & $1.02,1.14$ & 0.007 \\
\hline Fasting blood glucose serum concentration (mmol/L) & 1.11 & $1.04,1.18$ & 0.002 \\
\hline \multicolumn{2}{|c|}{ Using "estimated cerebrospinal fluid pressure" instead of blood pressure and body mass index: } \\
\hline Estimated cerebrospinal fluid pressure (mm Hg) & 1.22 & $1.13,1.31$ & $<0.001$ \\
\hline Age (years) & 1.11 & $1.08,1.13$ & $<0.001$ \\
\hline Fasting blood glucose serum concentration (mmol/L) & 1.11 & $1.04,1.18$ & $<0.001$ \\
\hline
\end{tabular}

independent parameters due to collinearity (lens thickness, anterior chamber depth, diastolic blood pressure, lowdensity lipoprotein serum concentration), and due to a lack of statistical significance, we dropped the parameters of axial length, uric acid serum concentration, blood platelet count, history of diabetes and hypertension, gender, waist-hip ratio and serum concentration of highdensity lipoprotein and albumin. In the final model, a higher prevalence of RVO originating at the optic disc was associated with older age $(P=0.005)$ and higher systolic blood pressure $(P=0.045)$. If the parameters of blood pressure and BMI were replaced by the eCSFP parameter, a higher prevalence of RVOs originating at the optic disc was associated in the final model with a higher eCSFP $(P=0.004)$ and older age $(P<0.001)$ (Table 5).

Compared with participants without RVOs, participants with RVO originating at arterio-venous crossings outside of the optic disc had a significantly (all $P<0.05$ ) older age, higher prevalence of men, shorter axial length, lower prevalence of diabetes and hypertension, higher systolic and diastolic blood pressure, higher BMI, higher fasting blood glucose serum concentration, and lower serum concentration of low-density lipoprotein and albumin, lower blood platelet count and higher waist-hip ratio (Table 4). In multivariable analysis, a higher prevalence of RVO originating outside of the optic disc was associated with older age $(P<0.001)$, higher systolic blood pressure $(P=0.009)$, higher BMI $(P=0.02)$, and higher fasting blood glucose serum concentration $(P<0.001)$ (Table 5). If the parameters of blood pressure and BMI were replaced by the eCSFP parameter, a higher prevalence of RVOs originating outside of the optic disc was associated in the final model with a higher eCSFP
$(P<0.001)$, older age $(P<0.001)$ and higher fasting blood glucose serum concentration $(P<0.001)$ (Table 5).

\section{Discussion}

In this cross-sectional examination of a community-based recruited Chinese population, the overall prevalence of any RVO was $0.96 \%$ ( $95 \%$ CI: $0.80,1.15)$. The RVO prevalence increased with older age, with participants aged $80+$ years having an approximately five times higher RVO prevalence than individuals aged under 60 years. ${ }^{8,9,19-22}$ A higher prevalence of RVO was associated with older age, higher eCSFP, and higher fasting glucose serum concentration. Similar relationships were found when the RVOs were stratified based upon the location of their origin.

The prevalence figures found for our study population agree with those reported for other populations. The Blue Mountains Eye Study in Australia and the North American Beaver Dam Eye Study reported RVO prevalences of 1.6\% and $0.8 \%$, respectively. ${ }^{21,22}$ Population-based studies on non-white populations aged $40+$ years revealed RVO prevalences of $0.7 \%$ in the Singapore Malay Eye Study, 2.1\% in the Japanese Hisayama Study, $1.2 \%$ in the Beijing Eye Study, $0.7 \%$ in the Central India Eye and Medical Study, and $0.72 \%$ in the Singapore Epidemiology of Eye Disease Study. ${ }^{7-10,19}$

The association between a higher RVO prevalence and higher eCSFP agrees with a previous investigation on smaller study populations in which the prevalence and incidence of RVOs correlated with higher eCSFP. ${ }^{12}$ In the longitudinal population-based Beijing Eye Study, a higher incidence of RVOs as a whole, and a higher incidence of CRVOs together with BRVOs originating at 
Table 4 Differences Between the Group of Participants with Retinal Vein Occlusion Originating at Optic Disc and Retinal Vein Occlusion Originating Outside Optic Disc Compared with the Group of Participants without Retinal Vein Occlusion in the Kailuan Eye Study

\begin{tabular}{|c|c|c|c|c|c|}
\hline & $\begin{array}{c}\text { No Retinal } \\
\text { Vein } \\
\text { Occlusions }\end{array}$ & $\begin{array}{l}\text { Vein } \\
\text { Occlusion } \\
\text { Starting } \\
\text { Within the } \\
\text { Optic Disc }\end{array}$ & $P$-value* & $\begin{array}{c}\text { Vein Occlusion Starting at } \\
\text { a Arterio-Venous Crossing } \\
\text { Outside of the Optic Disc } \\
\text { Vessel }\end{array}$ & $P$-value* \\
\hline Age (Years) & $52.9 \pm 13.1$ & $62.1 \pm 10.2$ & $<0.001$ & $61.0 \pm 9.7$ & $<0.001$ \\
\hline Gender (men) & $8386(67.79 \%)$ & & 0.17 & & 0.008 \\
\hline History of diabetes mellitus & 2297 (19.77\%) & & 0.53 & & $<0.001$ \\
\hline History of hypertension & $5308(45.70 \%)$ & & 0.009 & & $<0.001$ \\
\hline Mean systolic blood pressure $(\mathrm{mmHg})$ & $137.2 \pm 18.9$ & $150.0 \pm 20.6$ & $<0.001$ & $150.5 \pm 22.4$ & $<0.001$ \\
\hline Mean diastolic blood pressure $(\mathrm{mmHg})$ & $79.9 \pm 10.3$ & $88.1 \pm 9.2$ & 0.001 & $84.9 \pm 12.2$ & $<0.001$ \\
\hline Mean fasting blood glucose (mmol/L) & $6.06 \pm 1.96$ & $6.46 \pm 3.31$ & 0.39 & $7.33 \pm 3.26$ & $<0.001$ \\
\hline Mean heart rate (beats/minute) & $75.6 \pm 11.2$ & $75.81 \pm 13.05$ & 0.33 & $75.52 \pm 11.93$ & 0.695 \\
\hline Mean waist-hip ratio & $0.89 \pm 0.08$ & $0.91 \pm 0.07$ & 0.22 & $0.92 \pm 0.09$ & $<0.001$ \\
\hline Mean body mass index $\left(\mathrm{kg} / \mathrm{m}^{2}\right)$ & $25.0 \pm 3.4$ & $25.9 \pm 3.1$ & 0.16 & $26.1 \pm 3.8$ & 0.003 \\
\hline $\begin{array}{l}\text { Mean high-density lipoprotein cholesterol } \\
\text { concentration }(\mathrm{mmol} / \mathrm{L})\end{array}$ & $1.54 \pm 0.60$ & $1.44 \pm 0.41$ & 0.10 & $1.47 \pm 0.50$ & 0.399 \\
\hline $\begin{array}{l}\text { Mean low-density lipoprotein cholesterol } \\
\text { concentration }(\mathrm{mmol} / \mathrm{L})\end{array}$ & $2.97 \pm 0.92$ & $3.02 \pm 0.56$ & 0.65 & $2.78 \pm 0.83$ & 0.016 \\
\hline Mean triglyceride $(\mathrm{mmol} / \mathrm{L})$ & $2.10 \pm 2.26$ & $2.37 \pm 1.70$ & 0.81 & $2.24 \pm 1.74$ & 0.477 \\
\hline Mean total cholesterol concentration $(\mathrm{mmol} / \mathrm{L})$ & $4.63 \pm 1.97$ & $4.43 \pm 1.56$ & 0.72 & $4.39 \pm 1.75$ & 0.185 \\
\hline Mean uric acid concentration $(\mu \mathrm{mol} / \mathrm{L})$ & $326 \pm 88$ & $357 \pm 73$ & 0.02 & $346 \pm 109$ & 0.069 \\
\hline $\begin{array}{l}\text { Mean hypersensitive C-reactive protein } \\
\text { concentration }(\mathrm{mg} / \mathrm{L})\end{array}$ & $1.98 \pm 7.22$ & $2.37 \pm 2.72$ & 0.94 & $1.70 \pm 2.38$ & 0.722 \\
\hline Mean total protein concentration $(\mathrm{g} / \mathrm{L})$ & $73.2 \pm 5.1$ & $74.7 \pm 3.8$ & 0.20 & $73.9 \pm 5.6$ & 0.189 \\
\hline Mean albumin $(g / L)$ & $47.4 \pm 7.3$ & $46.6 \pm 2.4$ & 0.60 & $46.2 \pm 3.7$ & 0.001 \\
\hline Mean glutamate pyruvate transaminase $(\mathrm{U} / \mathrm{L})$ & $23.7 \pm 21.9$ & $21.8 \pm 17.1$ & 0.67 & $23.4 \pm 15.4$ & 0.916 \\
\hline Mean total bilirubin $(\mu \mathrm{mol} / \mathrm{L})$ & $15.2 \pm 12.0$ & $15.9 \pm 4.0$ & 0.59 & $15.6 \pm 6.4$ & 0.864 \\
\hline Creatinine $(\mu \mathrm{mol} / \mathrm{L})$ & $72.7 \pm 27.8$ & $79.1 \pm 19.3$ & 0.27 & $76.9 \pm 20.5$ & 0.171 \\
\hline Mean urea $(\mathrm{mmol} / \mathrm{L})$ & $5.65 \pm 5.99$ & $5.82 \pm 1.40$ & 0.83 & $5.89 \pm 1.59$ & 0.733 \\
\hline Mean urine $\mathrm{pH}$ & $5.87 \pm 0.62$ & $5.83 \pm 0.54$ & 0.50 & $5.94 \pm 0.71$ & 0.198 \\
\hline Mean red blood cell count $\left(10^{12} / \mathrm{L}\right)$ & $4.88 \pm 0.47$ & $5.07 \pm 0.42$ & 0.24 & $4.91 \pm 0.47$ & 0.430 \\
\hline Mean white blood cell count (109/L) & $5.93 \pm 1.77$ & $6.34 \pm 1.63$ & 0.27 & $6.05 \pm 1.98$ & 0.571 \\
\hline Mean blood platelet count $\left(10^{9} / \mathrm{L}\right)$ & $238 \pm 57$ & $241 \pm 46$ & 0.93 & $226 \pm 55$ & 0.038 \\
\hline Mean hemoglobin $(g / L)$ & $148.4 \pm 16.0$ & $154.1 \pm 15.3$ & 0.73 & $147.1 \pm 19.8$ & 0.998 \\
\hline Mean neutrophil cell count $\left(10^{9} / \mathrm{L}\right)$ & $3.73 \pm 2.08$ & $3.85 \pm 1.06$ & 0.54 & $3.87 \pm 1.44$ & 0.614 \\
\hline
\end{tabular}

(Continued) 
Table 4 (Continued).

\begin{tabular}{|l|c|c|c|c|c|}
\hline & $\begin{array}{c}\text { No Retinal } \\
\text { Vein } \\
\text { Occlusions }\end{array}$ & $\begin{array}{c}\text { Vein } \\
\text { Occlusion } \\
\text { Starting } \\
\text { Within the } \\
\text { Optic Disc }\end{array}$ & P-value* & $\begin{array}{c}\text { Vein Occlusion Starting at } \\
\text { a Arterio-Venous Crossing } \\
\text { Outside of the Optic Disc } \\
\text { Vessel }\end{array}$ & $\begin{array}{c}P \text {-value* } \\
\text { Central cornea thickness }(\mu \mathrm{m})\end{array}$ \\
\hline Anterior chamber depth $(\mathrm{mm})$ & $2.73 \pm 0.45$ & $2.47 \pm 0.50$ & 0.04 & $538 \pm 30$ & 0.853 \\
\hline Lens thickness $(\mathrm{mm})$ & $4.26 \pm 0.6 \mathrm{I}$ & $4.54 \pm 0.47$ & 0.07 & $4.40 \pm 0.36$ & 0.182 \\
\hline Axial length $(\mathrm{mm})$ & $23.71 \pm 1.28$ & $23.23 \pm 1.07$ & 0.44 & $23.45 \pm 0.89$ & 0.096 \\
\hline CSFP & $14.46 \pm 3.27$ & $14.45 \pm 2.34$ & 0.70 & $14.10 \pm 3.30$ & 0.048 \\
\hline
\end{tabular}

Notes: P-value*: statistical significance of the difference between the group with a retinal vein occlusion and the group without a retinal vein occlusion.

the optic nerve head, correlated with a higher eCSFP in multivariable analysis. ${ }^{22}$ These observations agree with the findings made in previous anatomical and physiological studies of the optic nerve head underlining the potential importance of the orbital CSFP. The latter is the counter pressure against the intraocular pressure (IOP) across the lamina cribrosa of the optic nerve head and one of the two determinants of the trans-lamina cribrosa pressure difference (TLCPD). ${ }^{23-29}$ The TLCPD is a main etiological parameter for pressure-related disorders of the optic nerve head region, including glaucomatous optic neuropathy with an abnormally high TLCPD and including papilledema with an abnormally low TLCPD. ${ }^{23-30}$ Since the central retinal vein passes through the space of the orbital CSFP, the intraocular central retinal vein pressure should be at least as high as the orbital CSFP is. This notion has been supported by experimental findings and by ophthalmodynamometric measurements of the central retinal vein pressure in patients with increased CSFP in whom an elevated central CSFP was associated with elevated ophthalmodynamometric measurements of the central retinal vein pressure. ${ }^{31-33}$

The association between a higher RVO prevalence and higher eCSFP may answer the question, why an increased blood pressure on the arterial side should lead to an increased venous outflow resistance out of the eye. The RVO-associated increase in the outflow resistance of the venous blood out of the eye leads to an increase in the intraocular venous pressure with subsequent venous congestion and venous dilatation inside of the eye, elevated capillary pressure and increased trans-capillary wall exudation and hemorrhage. Since arterial hypertension is directly related to the CSFP, an elevated arterial blood pressure may lead through the primarily elevated CSFP to a secondarily increased retinal vein pressure and an increased prevalence of RVOs. In an alternative manner, one can discuss that arterial hypertension leads to atherosclerosis which, due to a thickening and stiffening of the wall of the central retinal artery may lead to a compression or stenosis of the central retinal vein when passing through the central part of the lamina cribrosa. In a same manner, one may discuss that a stiffening of the retinal arteriolar wall may lead to a compression of the retinal venule at retinal arteriovenous crossing sites in eyes with BRVO at such crossing sites. One may consider in that context that the retinal arterioles, in contrast to arteries, do not have a continuous muscular coat (tunica muscularis), and thus show principal anatomical differences to arteries such as the central retinal artery. Fitting with the notion of an elevated CSFP as etiologic risk factor for RVOs are observations, that RVOs are usually noticed in the morning, and that macular edema due to RVOs is usually more marked in the morning than in the evening. ${ }^{34}$ The supine position during sleeping at night leads to an increased CSFP due to hydrostatic reasons. In agreement with this observation, the choroid is thinner in the afternoon than in the morning. ${ }^{35}$ Interestingly, the central retinal vein and the choroidal vortex veins drain their blood through the superior orbital vein into the intracranial cavity. The observations of diurnal changes in macular edema in patients with RVOs and of diurnal changes in choroidal thickness thus fit with a potential influence, the CSFP may have for the etiology of RVOs. 
Table 5 Associations (Multivariable Multinomial Logistic Regression Analysis) Between the Prevalence of Retinal Vein Occlusions Occurring in Different Location and Systemic Parameters in the Kailuan Eye Study

\begin{tabular}{|c|c|c|c|}
\hline \multicolumn{4}{|c|}{ Retinal Vein Occlusion Occurring Within Optic Disc } \\
\hline Parameters & Odds Ratio (OR) & 95\% Confidence Interval for OR & $P$-value \\
\hline Age (years) & 1.06 & $1.02,1.11$ & 0.005 \\
\hline Systolic blood pressure $(\mathrm{mm} \mathrm{Hg})$ & 1.02 & $1.00,1.05$ & 0.045 \\
\hline Body mass index $\left(\mathrm{kg} / \mathrm{m}^{2}\right)$ & 1.08 & $0.96,1.22$ & 0.203 \\
\hline Fasting blood glucose serum concentration (mM) & 0.97 & $0.78,1.19$ & 0.744 \\
\hline \multicolumn{4}{|c|}{ Using "estimated cerebrospinal fluid pressure" instead of blood pressure and body mass index: } \\
\hline Estimated cerebrospinal fluid pressure $(\mathrm{mm} \mathrm{Hg})$ & 1.26 & $1.08,1.48$ & 0.004 \\
\hline Age (years) & 1.13 & $1.08,1.19$ & $<0.001$ \\
\hline \multicolumn{4}{|c|}{ Retinal Vein Occlusions Occurring at Arterio-Venous Crossings Outside of the Optic Disc } \\
\hline Parameters & Odds Ratio (OR) & 95\% Confidence Interval for OR & $P$ value \\
\hline Age (years) & 1.05 & $1.03,1.07$ & $<0.001$ \\
\hline Systolic blood pressure $(\mathrm{mm} \mathrm{Hg})$ & 1.01 & $1.004,1.03$ & 0.009 \\
\hline Body mass index $\left(\mathrm{kg} / \mathrm{m}^{2}\right)$ & 1.08 & I.0I, I.14 & 0.016 \\
\hline Fasting blood glucose serum concentration (mM) & 1.13 & $1.06,1.21$ & $<0.001$ \\
\hline \multicolumn{4}{|c|}{ Using "estimated cerebrospinal fluid pressure" instead of blood pressure and body mass index: } \\
\hline Estimated cerebrospinal fluid pressure $(\mathrm{mm} \mathrm{Hg})$ & 1.20 & $1.11,1.31$ & $<0.001$ \\
\hline Age (years) & 1.10 & $1.07,1.13$ & $<0.001$ \\
\hline Fasting blood glucose serum concentration ( $\mathrm{mmol} / \mathrm{L})$ & 1.14 & $1.07,1.21$ & 0.001 \\
\hline
\end{tabular}

The association between CSFP and RVO prevalence as observed in the present and previous investigations sheds light on the general importance of the CSFP for the physiology and pathophysiology of the eye and optic nerve. Other examples are that choroidal thickness is positively correlated with the eCSFP and that the prevalence, incidence and severity of diabetic retinopathy was associated with the eCSFP. ${ }^{36,37}$ One may speculate whether the therapeutic reduction of the CSFP by oral application of carbonic anhydrase inhibitors may be worth to be evaluated as therapy of RVOs.

When the results of our study are discussed, its limitations should be taken into account. First, we used two photographic fields per eye to assess the prevalence of RVOs. It could have resulted in an underestimation of the prevalence of RVO if peripheral lesions might have been overlooked. Second, since the study had a crosssectional design, it could analyze only associated factors, while the detection of causative or risk factors needed a longitudinal study design. Third, the eCSFP was derived from a multivariate formula incorporating body mass index, diastolic blood pressure, and age. Although the eCSFP correlated relatively well with measured CSFP values in a previous study, one must bear in mind that the eCSFP value were just rough approximations needing further validation. ${ }^{38}$ In that context, it has also to be considered that the width of the orbital cerebrospinal fluid space was not taken into account, since it was not feasible to measure it in a large community-based investigation. Since a wider orbital cerebrospinal fluid space is associated with higher CSFP and has also been taken as a surrogate for CSFP, one may think however that the parameter of CSFP already contains the orbital cerebrospinal fluid space width as parameter. ${ }^{16}$ One may also discuss that the RVO prevalence in our study was primarily associated with higher blood pressure and elevated BMI, which were then put together in the formula for the estimation of the eCSFP. Due to anatomic reasons pointed out 
above, it makes however sense to discuss that it is primarily the CFSP, based on blood pressure and obesity, which influence the prevalence, and potentially the development, of RVOs. Fourth, some systemic parameters such as hyper-homocysteinemia and smoking, and some ocular parameters like intraocular pressure, were not examined in our study as potential factors associated with the prevalence of RVOs. ${ }^{6}$ Fifth, RVO is not one disease but it consists of CRVO, hemicentral RVO and branch BRVO, additionally differentiated into ischemic types versus nonischemic types. We did not differentiate between the ischemic type and the non-ischemic type of RVOs. One may also consider that patients with an ischemic RVO may have a lower life expectancy leading to a survival bias with an overestimation of the prevalence of non-ischemic RVOs in cross-sectional studies as ours. Sixth, we did not assess the know association between the prevalence of RVOs and glaucoma in our study. Seventh, due to the relatively small number of patients affected with RVOs, not all four types of RVO as described by Beaumont and Kang were differentiated in our study. ${ }^{39}$ Eighth, due to the inherent disadvantage of any population-based or community-based study, the number of individuals affected by the disease, ie, RVOs, was relatively small $(n=120)$ for a detailed multivariable analysis.

\section{Conclusion}

In this adult Chinese population, the prevalence of any RVO (mean: 0.96) was associated with older age, higher eCSFP and higher fasting serum blood glucose concentration. An elevated CSFP may play an etiologic role in RVOs.

\section{Acknowledgments}

Supported by the National Natural Science Foundation of China (81570891); Beijing Natural Science Foundation (7151003); the Beijing Municipal Administration of Hospitals' Ascent Plan (DFL20150201); Advanced Health Care Professionals Development Project of Beijing Municipal Health Bureau (2014-2-003); The Capital Health Research and Development of Special (2016-1-2051); Beijing Municipal Administration of Hospitals Clinical Medicine Development of Special Funding Support (ZYLX201307); Science \& Technology Project of Beijing Municipal Science \& Technology Commission (Z181100001818003, Z151100001615052); The Priming Scientific Research Foundation for the Junior Research in Beijing Tongren Hospital Capital
Medical University (2017-YJJ-ZZL-009 and NO. 2018YJJ-ZZL-046); Beijing Tongren Hospital Top Talent Training Program; 2018 Beijing Outstanding Young Talents, and Beijing Municipal Administration of Hospitals Incubating Program (Code: PX2019008).

\section{Disclosure}

The author reports no conflicts of interest in this work.

\section{References}

1. Rogers S, McIntosh RL, Cheung N, et al. International Eye Disease Consortium. The prevalence of retinal vein occlusion: pooled data from population studies from the United States, Europe, Asia, and Australia. Ophthalmology. 2010;117:313-319. doi:10.1016/j. ophtha.2009.07.017

2. Song P, Xu Y, Zha M, Zhang Y, Rudan I. Global epidemiology of retinal vein occlusion: a systematic review and meta-analysis of prevalence, incidence, and risk factors. J Glob Health. 2019;9 (1):1-10. doi:10.7189/jogh.09.010427

3. Hayreh S. Prevalent misconceptions about acute retinal vascular occlusive disorders. Prog Retin Eye Res. 2005;24(4):493-519. doi:10.1016/j.preteyeres.2004.12.001

4. McIntosh RL, Rogers SL, Lim L, et al. Natural history of central retinal vein occlusion: an evidence-based systematic review. Ophthalmology. 2010;117:1113-1123.

5. Rogers SL, McIntosh RL, Lim L, et al. Natural history of branch retinal vein occlusion: an evidence-based systematic review. Ophthalmology. 2010;117:1094-1101. doi:10.1016/j. ophtha.2010.01.058

6. Hayreh SS, Zimmerman B, McCarthy MJ, Podhajsky P. Systemic diseases associated with various types of retinal vein occlusion. Am J Ophthalmol. 2001;131:61-77. doi:10.1016/S0002-9394(00)00709-1

7. Lim LL, Cheung N, Wang JJ, et al. Prevalence and risk factors of retinal vein occlusion in an Asian population. $\mathrm{Br} J$ Ophthalmol. 2008;92:1316-1319. doi:10.1136/bjo.2008.140640

8. Liu W, Xu L, Jonas JB. Vein occlusion in Chinese subjects. Ophthalmology. 2007;114:1795-1796. doi:10.1016/j. ophtha.2007.03.010

9. Yasuda M, Kiyohara Y, Arakawa S, et al. Prevalence and systemic risk factors for retinal vein occlusion in a general Japanese population: the Hisayama study. Invest Ophthalmol Vis Sci. 2010;51:3205-3209. doi:10.1167/iovs.09-4453

10. Koh V, Cheung CY, Li X, et al. Retinal vein occlusion in a multi-ethnic Asian population: the Singapore Epidemiology of Eye Disease study. Ophthalmic Epidemiol. 2016;23:6-13. doi:10.3109/ 09286586.2015.1082604

11. Shin YU, Cho H, Kim JM, et al. Prevalence and associated factors of retinal vein occlusion in the Korean National Health and Nutritional Examination Survey, 2008-2012: a cross-sectional observational study. Medicine (Baltimore). 2016;95(44):e5185. doi:10.1097/ MD.0000000000005185

12. Jonas JB, Wang N, Wang YX, et al. Incident retinal vein occlusions and estimated cerebrospinal fluid pressure. The Beijing Eye Study. Acta Ophthalmol. 2015;93:e522-e526. doi:10.1111/aos.12575

13. Wu S, Huang Z, Yang X, et al. Prevalence of ideal cardiovascular health and its relationship with the 4-year cardiovascular events in the northern Chinese industrial city. Circ Cardiovasc Qual Outcomes. 2012;5:487-493. doi:10.1161/CIRCOUTCOMES.111.963694

14. Wang L, Cui L, Wang Y, et al. Resting heart rate and the risk of developing impaired fasting glucose and diabetes: the Kailuan Prospective Study. Int J Epidemiol. 2015;44:689-699. doi:10.1093/ ije/dyv079 
15. Xue H, Wang J, Hou J, et al. Prehypertension and chronic kidney disease in Chinese population: four-year follow-up study. PLoS One. 2015;10:e0144438. doi:10.1371/journal.pone.0144438

16. Xie $\mathrm{X}$, Zhang $\mathrm{X}, \mathrm{Fu} \mathrm{J}$, et al. Noninvasive intracranial pressure estimation by orbital subarachnoid space measurement: the Beijing Intracranial and Intraocular Pressure (iCOP) study. Crit Care. 2013;17:R162. doi:10.1186/cc12841

17. Hayreh SS, Zimmerman MB. Fundus changes in branch retinal vein occlusion. Retina. 2015;35:1016-1027. doi:10.1097/ IAE.0000000000000418

18. Hayreh SS, Zimmerman MB. Fundus changes in central retinal vein occlusion. Retina. 2015;35:29-42. doi:10.1097/ IAE.0000000000000256

19. Jonas JB, Nangia V, Khare A, Sinha A, Lambat S. Prevalence and associations of retinal vein occlusions: the Central India Eye and Medical Study. Retina. 2013;33:152-159. doi:10.1097/ IAE.0b013e318260246f

20. Keel S, Xie J, Foreman J, van Wijngaarden P, Taylor HR, Dirani M. Prevalence of retinal vein occlusion in the Australian National Eye Health Survey. Clin Experiment Ophthalmol. 2018;46:260-265. doi:10.1111/ceo.13031

21. Klein R, Klein BE, Moss SE, Meuer SM. The epidemiology of retinal vein occlusion: the Beaver Dam Eye Study. Trans Am Ophthalmol Soc. 2000;98:133-141.

22. Mitchell P, Smith W, Chang A. Prevalence and associations of retinal vein occlusion in Australia. The Blue Mountains Eye Study. Arch Ophthalmol. 1996;114:1243-1247. doi:10.1001/ archopht.1996.01100140443012

23. Berdahl JP, Allingham RR, Johnson DH. Cerebrospinal fluid pressure is decreased in primary open-angle glaucoma. Ophthalmology. 2008;115:763-768. doi:10.1016/j.ophtha.2008.01.013

24. Burgoyne CF, Downs JC, Bellezza AJ, Suh JK, Hart RT. The optic nerve head as a biomechanical structure: a new paradigm for understanding the role of IOP-related stress and strain in the pathophysiology of glaucomatous optic nerve head damage. Prog Retin Eye Res. 2005;24:39-73. doi:10.1016/j.preteyeres.2004.06.001

25. Jonas JB, Berenshtein E, Holbach L. Anatomic relationship between lamina cribrosa, intraocular space, and cerebrospinal fluid space. Invest Ophthalmol Vis Sci. 2003;44:5189-5195. doi:10.1167/ iovs.03-0174

26. Morgan WH, Yu DY, Cooper RL, Alder VA, Cringle SJ, Constable IJ. The influence of cerebrospinal fluid pressure on the lamina cribrosa tissue pressure gradient. Invest Ophthalmol Vis Sci. 1995;36:1163-1172.
27. Morgan WH, Yu DY, Alder VA, et al. The correlation between cerebrospinal fluid pressure and retrolaminar tissue pressure. Invest Ophthalmol Vis Sci. 1998;39:1419-1428.

28. Ren R, Jonas JB, Tian G, et al. Cerebrospinal fluid pressure in glaucoma. A prospective study. Ophthalmology. 2010;117:259-266. doi:10.1016/j.ophtha.2009.06.058

29. Volkov VV. Essential element of the glaucomatous process neglected in clinical practice. Oftalmol Zh. 1976;31:500-504.

30. Hayreh SS. Pathogenesis of optic disc edema in raised intracranial pressure. Prog Retin Eye Res. 2016;50:108-144. doi:10.1016/j. preteyeres.2015.10.001

31. Hayreh SS, Edwards J. Ophthalmic arterial and venous pressures. Effects of acute intracranial hypertension. $\mathrm{Br} J$ Ophthalmol. 1981;55:649-663. doi:10.1136/bjo.55.10.649

32. Jonas JB, Harder B. Ophthalmodynamometric estimation of cerebrospinal fluid pressure in pseudotumor cerebri. $\mathrm{Br} J$ Ophthalmol. 2003;87:361-362. doi:10.1136/bjo.87.3.361-a

33. Jonas JB, Pfeil K, Chatzikonstantinou A, Rensch F. Ophthalmodynamometric measurement of central retinal vein pressure as surrogate of intracranial pressure in idiopathic intracranial hypertension. Graefes Arch Clin Ophthalmol. 2008;246:1059-1060. doi:10.1007/s00417-008-0780-0

34. Paques M, Massin P, Sahel JA, et al. Circadian fluctuations of macular edema in patients with morning vision blurring: correlation with arterial pressure and effect of light deprivation. Invest Ophthalmol Vis Sci. 2005;46:4707-4711. doi:10.1167/iovs.05-0638

35. Gupta B, Grewal J, Adewoyin T, Pelosini L, Williamson TH. Diurnal variation of macular oedema in CRVO: prospective study. Graefes Arch Clin Exp Ophthalmol. 2009;247:593-596.

36. Jonas JB, Wang N, Wang YX, et al. Subfoveal choroidal thickness and cerebrospinal fluid pressure. The Beijing Eye Study 2011. Invest Ophthalmol Vis Sci. 2014;55:1292-1298.

37. Jonas JB, Wang $\mathrm{N}, \mathrm{Xu} \mathrm{J}$, et al. Diabetic retinopathy and estimated cerebrospinal fluid pressure. The Beijing Eye Study 2011 PLoS One. 2014;9:e96273.

38. Fleischman D, Bicket AK, Stinnett SS, et al. Analysis of cerebrospinal fluid pressure estimation using formulae derived from clinical data. Invest Ophthalmol Vis Sci. 2016;57:5625-5630. doi:10.1167/ iovs.16-20119

39. Beaumont PE, Kang HK. Clinical characteristics of retinal venous occlusions occurring at different sites. $\mathrm{Br} J$ Ophthalmol. 2002;86:572-580. doi:10.1136/bjo.86.5.572
Eye and Brain

\section{Publish your work in this journal}

Eye and Brain is an international, peer-reviewed, open access journal focusing on clinical and experimental research in the field of neuroophthalmology. All aspects of patient care are addressed within the journal as well as basic research. Papers covering original research, basic science, clinical and epidemiological studies, reviews and

Submit your manuscript here: https://www.dovepress.com/eye-and-brain-journa evaluations, guidelines, expert opinion and commentary, case reports and extended reports are welcome. The manuscript management system is completely online and includes a very quick and fair peerreview system, which is all easy to use. Visit http://www.dovepress com/testimonials.php to read real quotes from published authors. 